\title{
Desafios e possibilidades dos profissionais de saúde no cuidado ao idoso dependente
}

\author{
Challenges and possibilities of health professionals \\ in the care of dependent older adults
}

\author{
Raimunda Magalhães da Silva (https://orcid.org/0000-0001-5353-7520) ${ }^{1}$ \\ Christina César Praça Brasil (https://orcid.org/0000-0002-7741-5349) ${ }^{1}$ \\ Indara Cavalcante Bezerra (https://orcid.org/0000-0003-0647-2490) ${ }^{2}$ \\ Maria do Livramento Fortes Figueiredo (https://orcid.org/0000-0003-4938-2807) ${ }^{3}$ \\ Míria Conceição Lavinas Santos (https://orcid.org/0000-0001-5896-3895) ${ }^{1}$ \\ Jonas Loiola Gonçalves (https://orcid.org/0000-0003-1015-9173) ${ }^{1}$ \\ Maria Helena de Agrela Gonçalves Jardim (https://orcid.org/0000-0001-7622-1449) ${ }^{4}$
}

${ }^{1}$ Programa de Pós-

Graduação em Saúde

Coletiva. Universidade de Fortaleza. Av. Washington Soares 1321, Edson Queiroz. 60811-905 Fortaleza CE Brasil.rmsilva@unifor.br. ${ }^{2}$ Mestrado Profissional em Gestão em Saúde, Universidade Estadual do Ceará. Fortaleza CE Brasil. ${ }^{3}$ Programa de Pós-

Graduação em Enfermagem Centro de Ciências da Saúde, Universidade Federal do Piauí. Teresina PI Brasil. ${ }^{4}$ Curso de Enfermagem, Universidade da Madeira. Ilha da Madeira Portugal.
Abstract This study investigates the challenges and possibilities of health professionals in managing care for dependent older adults in PHC. We employed a qualitative approach based on dialectical hermeneutic fusion. Interviews were carried out with 38 health professionals, from June to December 2019, in eight cities in the five Brazilian regions, which resulted in themes about the challenges of professionals in the management of care for older adults, the strategies used, and their suggestions for improving the quality of care for dependent older adults. Management failures, structural factors, access barriers, scarce supplies, disrupted network, and lack of security, were pointed out as hardships in directing health protection, prevention, and promotion actions. The applied strategies are diverse and conflicting and aim to articulate the multiprofessional teams, which involve the Family Health Strategy and specialists to implement care. Strengthening reception, encouraging home consultation, mobilizing support groups, and carrying out health education were among the participants' integrative practices. Due to the complex study object, it was evidenced that professionals recognize the shortcomings in the health services and face undesirable situations due to the lack of qualified personnel in the teams and the defective materials and transportation.

Key words Health care, Health personnel, Dependent older adults, Primary Health Care, Qualitative research
Resumo Investiga desafios e possibilidades de profissionais de saúde para a gestão do cuidado de idosos dependentes na Atenção Primária à Saúde. Recorreu-se à abordagem qualitativa fundamentada na fusão hermenêutica dialética. Efetivou-se entrevista com 38 profissionais de saúde, nos meses de junho a dezembro de 2019, em oito cidades das cinco regiões brasileiras. Dos resultados, emergiram temáticas sobre os desafios que os profissionais enfrentam na gestão do cuidado ao idoso, as estratégias por eles utilizadas e suas sugestões para elevar a qualidade do cuidado aos idosos que dependem deste. Falhas na gestão, fatores estruturais, barreiras de acesso, escassez de insumos, desarticulação da rede e falta de segurança foram apontados como dificuldades para direcionar as ações de proteção, prevenção e promoção da saúde. Fortalecer o acolhimento, estimular a consulta domiciliar, mobilizar grupos de apoio e realizar educação em saúde figuraram entre as práticas integrativas sugeridas pelos participantes. Os profissionais reconhecem as deficiências nos serviços de saúde e enfrentam situações indesejadas pela ausência de pessoal qualificado nas equipes, bem como em razão da insuficiência de materiais e de transporte.

Palavras-chave Assistência à saúde, Profissionais de saúde, Idosos dependentes, Atenção Primária à Saúde, Pesquisa qualitativa 


\section{Introdução}

O envelhecimento da população faz parte da realidade demográfica no Brasil e no mundo. A Organização Mundial da Saúde (OMS) alerta para o fato de que em 2050 o mundo terá dois bilhões de idosos e, nas Américas, o número de pessoas acima de 60 anos aumentará mais de três vezes nos próximos 30 anos, passando de oito para 30 milhões ${ }^{1,2}$. O Brasil será o sexto país com o maior quantitativo de pessoas idosas até 2025, alcançando $22,71 \%$ da população total em 2050.

Com o avanço da idade, surgem problemas crônicos de saúde que, muitas vezes, tornam a pessoa idosa dependente de cuidado, juntamente com desafios dos sistemas de saúde que demandam linhas de cuidado e redes de atenção mais resolutivas ${ }^{3}$.

Para promover a longevidade e vidas saudáveis, a Organização Mundial da Saúde (OMS) lidera a Década do Envelhecimento Saudável 2020-2030 - em consonância com os Objetivos de Desenvolvimento Sustentável ${ }^{4}$. Em paralelo ao envelhecimento, desponta a urgência do cuidado a esta população para prevenir demências e incapacidades físicas e mentais ${ }^{5}$.

O Ministério da Saúde desenvolve de 1994 até o momento a Política Nacional do Idoso ${ }^{6}$. Desde o ano de 2000, as Políticas intensificaram-se - Estatuto do Idoso ${ }^{7}$; Política Nacional de Saúde da Pessoa Idosa ${ }^{8}$; Política de Atenção Básica ${ }^{9}$; Plano de Ações Estratégicas para o Enfrentamento das Doenças Crônicas não Transmissíveis no período 2011-2022 ${ }^{10}$; Política Nacional do Envelhecimento Ativo ${ }^{11}$; Diretrizes para o Cuidado das Pessoas Idosas no Sistema Único de Saúde (SUS); e Orientações Técnicas para a Implementação da Linha de Cuidado para Atenção Integral à Saúde da Pessoa Idosa no SUS ${ }^{12}$.

$\mathrm{O}$ aumento da expectativa de vida e a fragmentação do cuidado nas redes assistenciais ao idoso constituem uma realidade que influencia na resolubilidade do sistema e desampara, principalmente, o idoso dependente, o qual recebe o cuidado no domicílio. Consoante a este fato, a OMS/OPAS ${ }^{2}$ exprimem que o envelhecimento associado à incapacidade aumentou $12,6 \%$ desde 2009. O relatório do Plano de Ação da OPAS sobre Saúde das Pessoas Idosas 2009-2018 evidenciou que os trabalhadores de saúde não estão preparados para atender às necessidades das pessoas idosas.

A gestão do cuidado em saúde deve envolver múltiplas dimensões, ajustando-se a cada fase da vida, em busca de bem-estar, segurança e auto- nomia, podendo ser beneficiada pelo acesso aos recursos de saúde disponíveis no meio social ${ }^{13}$. No âmbito organizacional do SUS, a Atenção Primária à Saúde (APS) representa a principal porta de entrada do sistema, pois é onde deve ocorrer o primeiro contato dos usuários com os serviços e os profissionais de saúde, de forma longitudinal, visando à integralidade do cuidado, com foco em ações de prevenção e promoção da saúde ${ }^{14}$. $\mathrm{Na}$ reorientação do modelo assistencial em saúde na APS, a Estratégia Saúde da Família (ESF) protagoniza a atenção voltada para a família e direcionada à comunidade ${ }^{14}$.

Na perspectiva de Oliveira et al. ${ }^{15}$, a maneira de cuidar do idoso no Brasil, ressalta-se aqui o dependente, deve ser repensada, de modo que haja um percurso assistencial lógico e coerente ante uma rede articulada de serviços e pessoas. Essas transformações devem ser implementadas nos serviços, na formação dos profissionais de saúde, no apoio à organização e na gestão dos recursos, bem como no financiamento.

Impõe-se, contudo, elucidar as estratégias utilizadas para a prevenção de riscos e o gerenciamento das condições de saúde dessa população. Acredita-se que entender os desafios dos profissionais que lidam cotidianamente com o cuidado a esses idosos dependentes no âmbito da Atenção Primária à Saúde (APS), investigar as estratégias de cuidado por eles empregadas e as sugestões de melhorias com vistas a apoiar o enfrentamento dos problemas de saúde dessa população fragilizada, pode abrir novos caminhos e fortalecer a integralidade do cuidado no SUS. Com efeito, objetivou-se investigar os desafios e as possibilidades de profissionais de saúde para a gestão do cuidado de idosos dependentes na Atenção Primária à Saúde.

\section{Método}

Este artigo é oriundo de um estudo multicêntrico denominado Estudo situacional dos idosos dependentes que residem com suas famílias visando a subsidiar uma politica de atenção e de apoio aos cuidadores, vinculado ao Departamento de Estudos sobre Violência e Saúde Jorge Careli da Fundação Oswaldo Cruz.

Realizou-se estudo qualitativo numa perspectiva hermenêutica-dialética, que ancora os processos compreensivos e críticos de um fenômeno social $^{16,17}$. A hermenêutica busca aproximar-se do significado estabelecido pelos profissionais, ao vivenciarem a experiência do cuidado com o 
idoso dependente, ressaltando crenças, valores, ações objetivas e sentidos subjetivos. A dialética possibilita o confronto entre os componentes sociais por meio de uma visão crítica.

O estudo ora relatado foi desenvolvido em oito municípios das cinco regiões do Brasil, incluindo as cidades de Brasília, Teresina, Fortaleza, Manaus, Rio de Janeiro, Belo Horizonte, Porto Alegre e Araranguá. A escolha desses municípios adveio da necessidade de conhecer as realidades do País, em razão da diversidade e complexidade que envolvem múltiplos aspectos - como as condições sociais, culturais e de saúde ${ }^{18}$.

Para este recorte do estudo, foram entrevistados 38 profissionais que atuavam na APS e realizavam algum atendimento com idosos dependentes. Dentre as categorias profissionais, foram entrevistados médico (8), enfermeiro (18), psicólogo (1), fisioterapeuta (1), assistente social (3), dentista (2), biólogo (1), educador físico (1), técnico de enfermagem (1), pedagogo (1) e agente comunitários de saúde (1). Para garantir a experiência em assistir idosos dependentes, incluíram-se partícipes com, no mínimo, um ano de atuação nos serviços da APS. Foram excluídos os que estavam de férias ou afastados por qualquer pretexto das atividades laborais.

Informantes-chaves das secretarias de saúde municipais indicaram os participantes, com base no conhecimento prévio sobre o cadastro de domicílios e UBS que atendiam pessoas idosas dependentes de qualquer tipo de cuidado, fosse físico, mental ou social. Os profissionais convidados foram contatados pessoalmente, por telefone ou mídias sociais. Ao aceitarem o convite, receberam informações sobre o estudo.

A coleta de dados ocorreu por meio de entrevista semiestruturada para captar dados de identificação, características sociodemográficas, facilidades e dificuldades para acolher e acompanhar o cuidado da pessoa idosa dependente, bem como estratégias de apoio utilizadas. A entrevista aconteceu após a assinatura do Termo de Consentimento Livre e Esclarecido (TCLE) pelo participante e realizada de junho a dezembro de 2019, em locais reservados nos ambientes de trabalho do respondente, com duração média de 20 minutos.

Os dados foram ordenados, classificados e analisados seguindo as etapas de transcrição das entrevistas, leitura, releitura do material e separação dos conjuntos de signos emergidos do texto (ordenação); agrupamento dos conjuntos de signos e classificação analítica destes (classificação), e interpretação dos dados, trazendo à tona as com- plementaridades, as convergências, as divergências e o inusitado, oriundos do universo empírico ${ }^{17}$.

A pesquisa obedece aos ditames da Resolução $466 / 2012^{19}$, havendo sido aprovada pelo Comitê de Ética e Pesquisa da Fundação Oswaldo Cruz. Para resguardar as identidades dos participantes, utilizaram-se as abreviaturas das profissões, seguidas do nome da cidade em que foi realizada a coleta.

\section{Resultados}

Os resultados estão apresentados em duas temáticas emergidas do material empírico coletado. Vale ressaltar que a alusão ao 'idoso' é extensiva ao 'idoso dependente' (ID), uma vez que os pesquisadores analistas deste estudo consideraram o princípio da indissociabilidade, já que pertencem ao mesmo grupo populacional, diferindo apenas quanto ao grau ou à intensidade de cuidado demandado.

\section{Desafios para o profissional na gestão do cuidado ao idoso dependente}

Os principais desafios relatados pelos participantes estão relacionados à gestão ineficiente da APS; dificuldades estruturais; barreiras territoriais e de acesso; escassez de insumos; burocracia no sistema de contrarreferência e filas de espera demoradas; e falta de segurança pública.

A gestão ineficiente da APS é revelada pela defasagem na atenção e que não tem prioridade de recursos e gerência.

A atenção básica, de básica não tem nada! Ela acaba ficando muito mais em consultórios, perdendo a capacidade importantíssima de identificação dentro da casa das pessoas. (Enf., Porto Alegre).

Quando se fala em atenção primária, a gente pensa em prevenção, educação em saúde. Hoje a gente está fazendo $1 \%$ de educação e saúde. O restante é só demanda espontânea. Então [as ações realizadas] perderam um pouco o sentido de atenção primária. (Med., Fortaleza).

As dificuldades estruturais na APS são desafiadoras e merecedoras de atenção pelos órgãos responsáveis. Os profissionais almejam espaços, para realizar atividades coletivas e formar uma rede de atenção compartilhada.

$\mathrm{Na}$ unidade de saúde, tem a sala do NASF que não comporta nem os profissionais do NASF. Se tiver 5 profissionais dentro da sala, fica lotada. Então, não tem como desenvolver grupos. (Psic., Fortaleza). 
Como eu posso implementar alguma coisa diferente sem ter um espaço para isso? Sem ter um profissional comigo? Nada é fácil para a atenção básica, nada é fácil! Criar uma rede é difícil! (Enf., Rio de Janeiro).

As dificuldades da assistência ao ID também existem na unidade de saúde, em razão das barreiras territoriais e de acesso:

Não são todas as unidades que têm um bom acesso, que têm elevador, por exemplo. Você vê aqui uma escada. A gente tentou colocar os clínicos lá embaixo, exames, a enfermagem, mas se uma idosa precisa ir ao ginecologista, tem que subir escada. (AS, Belo Horizonte).

O posto foi construído em uma área em que a demanda maior vem do outro lado da avenida. Isso ai é uma barreira para os idosos, porque não tem ônibus e eles não conseguem atravessar. (Enf., Fortaleza).

$O$ deslocamento é uma barreira. $O$ idoso tem que vir buscar o medicamento aqui, o idoso mais frágil não consegue vir aqui, sabe? (Med., Belo Horizonte).

Além de problemas de estrutura e de acesso dos ID aos serviços, os profissionais reconhecem as queixas dos familiares:

Ele tem muita dificuldade em levar o idoso para fazer qualquer tipo de exame, com exceção da coleta domiciliar, que também realizamos, mas, geralmente, eles não têm condições de levar o usuário a qualquer hospital para fazer uma simples radiografia. (Enf., Rio de Janeiro).

Nem sempre as casas são próximas ao posto. Quando são, a gente vai acompanhando o idoso. (Enf., Porto Alegre).

As particularidades de algumas cidades revelam a escassez de insumos para procedimentos simples e de biossegurança:

A gente tem dificuldade em ter material para tratar um ferimento. Às vezes leva um mês ou mais para o pedido chegar. Falta material básico: luvas, soro, material para curativo. (Enf., Teresina).

Exames de imagem e outros exames que não acontecem dentro da unidade básica e que o idoso tem que fazer, ainda demoram mais. Exames que precisam de encaminhamentos para especialistas, apresentam dificuldade ainda maior. (Enf., Fortaleza).

Outras dificuldades são as filas de espera demoradas e a burocracia no sistema de contrarreferência para o acompanhamento e/ou encaminhamento dos ID, o que prejudica a resolubilidade da assistência:

O paciente é cadastrado e tem uma fila de espera. Tem pessoas que ficam na fila vários meses, sem conseguir realizar os exames, nem a consulta com o especialista. Quando ele tem uma fragilidade maior e precisa da atenção secundária, a gente encaminha esse paciente e observa várias barreiras. (Med., Fortaleza).

Tem especialidade que a gente consegue acompanhar mais rapidamente, a exemplo da geriatria. Paciente com Alzheimer, a gente consegue encaminhar para o geriatra, já para a neurologia, demora. O encaminhamento fica aqui muito tempo. (Enf., Teresina).

A burocracia do SUS é muito grande, a regulação demora. O contingente de idosos é muito grande, a demora para atender todas as necessidades é grande. (Enf., Fortaleza).

A falta de segurança pública em diversos territórios expressa a realidade das metrópoles brasileiras.

O agente de saúde sempre vai acompanhando a enfermeira para não ir sozinha, por uma questão de segurança da área, a gente procura não fazer visitas domiciliares sozinhos. (Enf., Porto Alegre).

No território, às vezes, ninguém podia fazer a visita, porque estava acontecendo tiroteio ou algo que impedia ou dificultava essa visita. Várias vezes isso aconteceu. (Enf., Fortaleza).

\section{Estratégias e sugestões dos profissionais para o cuidado ao idoso dependente}

Comunicação interprofissional; contratação de profissionais e especialistas; acolhimento; grupos terapêuticos e comunitários; capacitação e ações de educação permanente com cuidadores e familiares; e visitas domiciliares foram estratégias apontadas como necessárias para o cuidado ao ID.

Os profissionais afirmaram que a atenção à saúde do idoso necessita de articulações interdisciplinares e multiprofissionais para a formação de uma rede de cuidado mais eficiente.

Para melhorar a qualidade de atendimento a esses idosos, é necessário reforçar a rede multiprofissional. (Med., Fortaleza).

Os NASF têm vários profissionais de reabilitação, como o assistente social, psicólogo, fisioterapeuta, nutricionista, farmacêutico, terapeuta ocupacional, e a gente usa essa rede para conversar com eles sobre as necessidades do idoso. (AS, Belo Horizonte).

A gente tem uma apoiadora que é da regional municipal de saúde. Ela faz uma revisão de toda a estrutura da ESF e procura sanar os problemas com relação à assistência dessas famílias. (Enf., Teresina). 
Os participantes perceberam a carência de recursos humanos em diversas áreas, destacando a necessidade de contratação de especialistas para fortalecer a assistência na ESF.

Para uma melhor atenção ao idoso é preciso ter na equipe mais geriatras, porque um só não consegue fazer muita coisa. (Med., Brasília).

$O$ assistente social viria para dar esclarecimento do que é o papel do cuidador, até onde ele pode ir, em que momento ele pode ajudar [...], mas a gente não tem o assistente social, faz muita falta. (Enf., Rio de Janeiro).

Se essa equipe fosse completa com mais profissionais, tipo psicólogo, para dar um suporte melhor para esses idosos, seria muito bom. (ACS, Manaus).

$\mathrm{Na}$ busca de estratégia resolutiva para os problemas cotidianos, evidenciou-se a intenção de acolher o ID no serviço de saúde.

Acolher é o mais fácil. Acolher, ouvir, a gente tem grupo, tem terapia, tem acolhimento todo dia que chega na demanda espontânea. Você senta, escuta e vê o que pode fazer. (Enf., Fortaleza).

As iniciativas que podem ser empregadas sempre devem ser pensadas no bem estar do paciente. $O$ acolhimento exerce papel fundamental neste processo, pois é o momento de criar vínculo com a família e com o paciente. (Fisio., Manaus).

Para promover a saúde da população de ID, os profissionais reconheceram os grupos comunitários como iniciativas favoráveis ao cuidado. Em alguns serviços de saúde, as práticas integrativas complementares são vistas como valiosas para esse público.

Um trabalho coordenado pelo serviço social uma vez por semana é focado no cuidador. Principalmente os familiares cuidadores de idosos dependentes recebem orientação e instrução. O mais interessante é a conversa entre eles, um ajudando o outro, é o momento de desabafar, é terapêutico. (Psic., Brasília).

A gente tem o grupo "As Empoderadas". São senhoras que fazem artesanato, viúvas, os filhos já saíram de casa, é muito potente porque você tira do ócio, da depressão, você dá a forma para essa mulher ganhar dinheiro, ela faz artesanato e vende, faz a sociabilização, viram amigas, é uma rede maravilhosa! (Enf., Rio de Janeiro).

Nessa unidade de apoio já existe terapia comunitária. Isso está expandido, e para o idoso é valioso! Aqui no posto a gente também tem uma terapia comunitária para o cuidador que, às vezes, está mais debilitado que o idoso. Ele está chorando, mais ansioso, devido ao cansaço de cuidar dele dia e noite. (Enf., Fortaleza).
Ações baseadas em práticas interdisciplinares partem dos profissionais para auxiliar os cuidadores e familiares de ID.

Uma das maiores iniciativas é um curso de cuidador, que, muitas vezes, é outro idoso. Algumas informações básicas, principalmente de higiene, manuseio dos alimentos, coisas que possam evitar que ele tenha alguma complicação, escaras. (Enf., Teresina).

Uma formação de cuidadores, especialmente, dos familiares com algumas técnicas de mobilidade no leito, de transferência, que eles podem utilizar no dia a dia. (To., Fortaleza).

A gente faz tanta educação na escola para falar de cuidado com criança, para falar com gestantes, grupo de pré-natal, a gente deveria ter também para o idoso, para a família saber que as necessidades são outras, que às vezes tem que ter alguns cuidados especiais. (Med., Belo Horizonte).

Os profissionais enfatizaram a visita domiciliar intermediada por ações de educação em saúde como recurso fundamental de cuidado, principalmente aos ID que não frequentam o posto de saúde ou vivem sozinhos.

Quando a gente vai fazer alguma visita, a gente tenta orientar as questões sobre alimentação, hidratação, cuidado com a pele, a questão dos tapetes (para evitar quedas), da dificuldade do idoso. (Enf., Porto Alegre).

A gente disponibiliza aqui orientações individuais. Quando a gente vai ao domicílio, a gente faz orientação à família sobre determinados cuidados com o idoso. (Enf., Teresina).

\section{Discussão}

Quanto aos desafios para o profissional ante o cuidado ao ID no âmbito da APS, a percepção dos profissionais evidenciou que a gestão dos serviços de saúde direcionados à essa população é ineficiente. Ravioli et al. ${ }^{20}$ analisaram a gestão de serviços no SUS, de 2005 a 2016, e detectaram dificuldades administrativas dos gestores, como a “[...] baixa capacidade operacional, falta de autonomia orçamentária, financeira e administrativa, baixos controles de qualidade, influência política externa, burocracia excessiva para contratar pessoal, efetuar adequações de estrutura e comprar insumos, medicamentos e equipamentos".

De um modo geral, as ações ineficientes da gestão desafiam os processos que constituem a APS, desde a contratação de recursos humanos, a organização de serviços e a aquisição de insu- 
mos. Os gestores públicos precisam conhecer seus problemas, estudar os indicadores de saúde, para que saibam planejar, estruturar, organizar e avaliar as ações de natureza técnico-científica e político-institucionais e estabelecer projetos que possam intervir sobre o estado de saúde da população ${ }^{21}$. Os gestores e os profissionais que prestam assistência à saúde devem repensar o trabalho realizado $^{22}$, tomando por base as necessidades dos usuários ${ }^{23}$.

Veras e Oliveira ${ }^{24}$, ao analisarem o modelo de atenção à saúde do idoso apontam que, nos sistemas de saúde atuais, os problemas da fragmentação do cuidado e da má coordenação poderão ser resolvidos com base em modelos de cuidados integrados. Para tanto, os autores sugerem o redesenho dos níveis de cuidado, que assegure um fluxo coerente de ações de educação, promoção da saúde e prevenção de problemas, criando uma linha de cuidado que privilegie o acolhimento ao idoso e o acompanhamento de cuidados paliativos.

A falta de espaço para desenvolver o trabalho com o ID também foi identificada na pesquisa sobre o Censo de Estrutura da Atenção Primária à Saúde no Brasil ${ }^{25}$, que pode estar relacionada ao uso de casas adaptadas para o funcionamento de unidades de saúde, o que, muitas vezes, ocorre sem um planejamento ou projeto específico.

As barreiras territoriais e a falta de acessibilidade aos serviços de saúde identificadas neste estudo refletem-se na cobertura assistencial, pois fragmentam o ciclo do cuidado, quebram a assiduidade dos usuários e fragilizam os vínculos com os profissionais ${ }^{26}$. No Censo realizado por Poças et al. ${ }^{25}$, as situações encontradas assemelham-se à inadequação da estrutura nos ambientes físicos, dificuldade de transporte e de acesso, principalmente de idosos e portadores de deficiências.

A escassez de insumos para procedimentos básicos figurou entre as dificuldades relacionadas à produção do cuidado na ESF nas diversas regiões do Brasil. A falta de materiais básicos para curativos em ID e acamados torna-se uma tarefa inexequível. Outros estudos ${ }^{25,27}$ demonstraram um problema macro de má gestão de recursos no País.

As múltiplas demandas relacionadas aos ID exigem ações intersetoriais de atenção à saúde. São apontados, no entanto, desafios na consolidação do cuidado integral à esse público, como a dificuldade de articulação com a rede de atenção e a burocratização para que o ID seja acolhido em toda sua complexidade ${ }^{14}$. Essa articulação favorece a integralidade do cuidado e deve ocorrer considerando-se todos os recursos do território, desde serviços de saúde, praças, igrejas, clubes, centros profissionalizantes e associações, com vistas a atender demandas afetivas, sanitárias, sociais, econômicas, culturais, religiosas, educacionais e de lazer para o idoso ${ }^{28}$.

A burocracia do sistema de referência e contrarreferência e das longas e demoradas filas de espera para atendimento aos ID nas especialidades médicas interrompe a continuidade do cuidado no percurso terapêutico. Schenker e Costa ${ }^{14}$ caracterizam como fragmentado e descontextualizado o encaminhamento para a atenção especializada, e isto fragiliza o cuidado ao ID.

Kantorski et al..$^{28}$ evidenciam que o sistema de referência e contrarreferência deve ser considerado um recurso importante na linha de cuidado, uma vez que, se bem estruturado, promove o fluxo eficiente de encaminhamento de usuários aos diversos níveis de atenção e recomenda a integração dos serviços de maneira comprometida e efetiva.

Outro fator é a falta de segurança pública para realizar as atividades, principalmente a visita domiciliar aos idosos. Sturbelle et al..$^{29}$ acentuam que a violência nos serviços de saúde aumenta dia a dia. Na APS, os serviços são desenvolvidos na própria comunidade, seja nas unidades de saúde, em outros espaços e nos próprios domicílios. Isto aproxima os profissionais do território, podendo expô-los à violência, especialmente nas zonas de tráfico de drogas. Essa situação tornase mais grave nas capitais, em consequência da urbanização, associada ao crescimento da criminalidade.

As polícias brasileiras têm dificuldades em conter a violência por depararem com o crescimento de facções (grupos criminosos organizados e bem equipados) e por suas precárias condições de trabalho para enfrentá-los. Souza e Minayo $^{30}$ assinalam que a solução não seria apenas fornecer mais armamentos à polícia, mas oferecer-lhes treinamento de excelência, apoio diário em áreas conflagradas, descanso adequado e amparo emocional - não esquecendo a necessidade de investimentos em infraestrutura na polícia e nos serviços de saúde em prol da segurança dos trabalhadores de saúde ${ }^{29}$.

No que diz respeito às estratégias e sugestões dos profissionais para o cuidado ao ID na APS, destaca-se o ensejo pela melhoria na articulação com a equipe interdisciplinar e multiprofissional, por uma abordagem centrada em uma assistência integral e contínua. As repercussões transpõem o modelo biomédico e configuram o envolvimento dos próprios usuários com a sua rede de apoio, 
trazendo corresponsabilização para o conjunto terapêutico do cuidado ${ }^{31}$.

$\mathrm{Na}$ China, pesquisadores evidenciam que o atendimento interdisciplinar é um benefício para melhorar o desempenho das condições crônicas do ID, exemplificando que pacientes com doença de Alzheimer têm ganhos funcionais significativos ante essa abordagem ${ }^{32}$.

A valorização da equipe multidisciplinar no âmbito da gestão do cuidado ao ID tem sido um grande desafio das políticas de saúde pública, sendo urgente uma atenção ampliada que considere estratégias de estruturação e reorganização dos serviços, além de modificações no processo de trabalho ${ }^{33}$. Nessa direção, os saberes e as tecnologias da clínica continuam sendo privilegiados, e podem juntar-se ao comprometimento e ao vínculo, desempenhando práticas mais integradas de intersetorialidade e interdisciplinaridade, a fim de ampliar e fortalecer o cotidiano das equipes da $\mathrm{ESF}^{34}$.

$\mathrm{Na}$ busca desse tipo de cuidado, os relatos emitem sugestões para efetivar os profissionais no serviço e realizar contratações de especialistas a fim de que atuem junto ao ID. A efetivação dos profissionais na rede de atenção à saúde é destacada como anseio dos participantes desta pesquisa, visto que a sua fixação consolida os vínculos terapêuticos com a população adscrita, ampliando o reconhecimento do território e fortalecendo as práticas em saúde, o que potencializa a resolubilidade da assistência à saúde do $\mathrm{ID}^{35,36}$.

Ressalta-se que, para efetivar um profissional, é necessário observar fatores como localização da unidade de saúde, idade do trabalhador, função, nível de especialidade, pressão e satisfação na atividade laboral. A não observância destes fatores pode levar à rotatividade, como destacado por 42,3\% dos médicos da APS em um estudo desenvolvido na cidade de Chongqing, na China ${ }^{37}$, cujos pesquisadores constataram a necessidade de reformulação dos incentivos e das propostas de trabalho para a fixação e a continuidade da assistência do profissional na APS ${ }^{37}$.

Nessa perspectiva, as contratações de especialistas nas áreas da Geriatria e Gerontologia podem prover e/ou consolidar uma assistência mais resolutiva ao ID, facilitando o trabalho no contexto das redes de atenção à saúde ${ }^{38,39}$.

$\mathrm{O}$ acolhimento foi destacado como recurso diversificado com múltiplas interfaces na saúde do ID e que podem interferir durante a efetivação do cuidado, sendo considerado um desafio para as equipes de saúde ${ }^{40}$. Sobre 'acolher', as falas dos profissionais vão ao encontro da Política
Nacional de Humanização, pois o acolhimento privilegia espaço para a escuta e recepção do ID e demais usuários ${ }^{41}$.

Neste estudo, a realização de grupos comunitários foi destacada como realidade nos serviços de saúde, partindo da iniciativa de vários profissionais. Em contrapartida, a ausência de grupos focados exclusivamente no ID também foi observada. A literatura destaca os grupos de apoio como métodos para ampliar o cuidado ao ID, com ênfase na prevenção e promoção da saúde, muito embora a participação dos idosos nesses grupos ocorra como aderência ao tratamento, quando já existe um problema de saúde ${ }^{14}$.

A saúde de idosos requer uma lógica na assistência do tipo rede, de integralidade do cuidado e nos aspectos de macro ou micro gestão. Para a implementação desses cuidados, compete à gestão realizar as articulações cabíveis para a composição da rede e do plano de cuidado prioritário ${ }^{42}$.

Relativamente ao cuidador familiar, é sensível a carência de apoio e orientação. Os profissionais revelaram o objetivo de intensificar a perspectiva para a família do idoso, por ser a principal provedora de cuidados. Araújo et al. ${ }^{43}$ postulam a noção de que as ações para empoderamento da família não se mostram suficientes e que se deve compreender o contexto familiar, de modo a auxiliar nas relações e no cuidado com o ID. As políticas públicas precisam promover o cuidado e a viabilização para que a família possa cuidar, observando como o idoso se vê no contexto familiar, o que interfere na execução de práticas que facilitem as atividades cotidianas.

Na gestão do cuidado, a família há que ser visualizada no contexto multidimensional, valorizando a singularidade das relações interpessoais familiares, dando sentido às vivências, experiências, religião e cultura ${ }^{33}$.

Destaca-se a ideia de que o fortalecimento da APS é considerado estratégia global. Na Tailândia, programas de capacitação desenvolvidos por uma década indicaram que, uma vez instituídas as múltiplas competências dos membros da APS, ampliou-se a resolubilidade de problemas complexos em distintos contextos locais, potencializando os resultados assistenciais das equipes de países de baixa e média renda ${ }^{44}$.

A visita domiciliar é divisada como excelente estratégia de cuidado para o ID na percepção dos profissionais. A consolidação dessa estratégia é destacada como apoiadora em estudo conduzido em comunidades rurais japonesas, nas quais alunos de Enfermagem difundem ações preventivas, 
englobando perspectivas individuais e ambientais de bem-estar na vida comunitária ${ }^{45}$.

Com efeito, esses achados corroboram o 'retrato' da atenção primária no Canadá, o qual destaca a potencialidade da assistência no contexto do domicílio, salientando as experiências aprimoradas para os pacientes e cuidadores. Esse fato leva à redução de custos com assistência médica, principalmente quando o cuidado é fornecido por equipes interprofissionais ampliadas ${ }^{46}$.

\section{Considerações finais}

O cuidado à pessoa idosa, associado a variados tipos de dependência decorrentes da própria longevidade, sejam estas sociais, físicas ou emocionais, exige das instituições e dos profissionais atitudes que determinem padrões assistenciais eficazes e promotores de uma prática habilitada a ascender a qualidade de saúde e de vida dos idosos e seus familiares. Essa prática deve estar ancorada no diálogo, na interrelação dos envolvidos e na inserção de ações inovadoras que viabilizem condições favoráveis para o engajamento das pessoas que proporcionam o cuidado.

A gestão do cuidado ao ID configura uma circunstância ímpar, ampla e complexa, na contextura da qual o operador de saúde assume dimensões desafiadoras para tomar decisões, envolvendo proteção, prevenção e promoção da saúde, priorizando recursos e a gerência do ser- viço. As condições estruturais e administrativas dos serviços de APS, das cidades sob estudo, demonstraram fragilidades na assistência ao ID no domicílio e na unidade de saúde, ressaltando a gestão ineficiente; dificuldades na área física, territoriais e de acesso; falta de material para o cuidado; burocracia administrativa no sistema de contrarreferência; filas de espera demoradas; e a falta de segurança pública.

Os profissionais revelaram que as dificuldades nos serviços de saúde estão relacionadas a diversos fatores, mas lutam para supri-las, empreendendo ações que primam por maior integração da equipe multiprofissional, adequações para melhorar o ambiente de trabalho, assim como o envolvimento dos recursos comunitários. Ressaltaram que os grupos comunitários, as práticas integrativas complementares e a educação em saúde são fundamentais para o desenvolvimento de ações protetivas para o ID e seus familiares.

No universo do cuidado ao idoso na APS, especialmente para aqueles dependentes de cuidado, os desafios enfrentados pelos profissionais são diversos e complexos, exigindo uma articulação interprofissional e intersetorial eficaz para a ampliação da resolubilidade. Desse modo, verifica-se a necessidade de um redimensionamento das práticas de saúde direcionadas aos idosos, considerando aspectos estruturais, administrativos, sociais e políticos, que promovam mudanças em condições essenciais para gerenciar a demanda diária, na unidade de saúde e nos domicílios.

\section{Colaboradores}

Nossa contribuição foi de concepção e planejamento do projeto de pesquisa (RM Silva, CCP Brasil e MHAG Jardim); coleta de dados, análise e interpretação dos resultados (IC Bezerra, JL Gonçalves, e MLF Figueiredo); redação do manuscrito (IC Bezerra, CCP Brasil, MCL Santos e RM Silva) e revisão crítica do manuscrito (RM Silva, CCP Brasil, JL Gonçalves e MLF Figueiredo). 


\section{Referências}

1. Organização Mundial da Saúde (OMS). Mundo terá 2 bilhões de idosos em 2050; OMS diz que 'envelhecer bem deve ser prioridade global'. Brasília: Organização Pan-Americana da Saúde; 2014 [acessado 2020 Abr 28]. Disponível em: https://nacoesunidas.org/mundo-tera-2-bilhoes-de-idosos-em-2050-oms-diz-queenvelhecer-bem-deve-ser-prioridade-global/

2. Organização Mundial da Saúde (OMS). Número de pessoas idosas com necessidade de cuidados prolongados triplicará nas Américas até 2050. Brasília: Organização Pan-Americana da Saúde; 2019 [acessado 2020 Abr 28]. Disponível em: https://nacoesunidas.org/numero-de-pessoas-idosas-com-necessidade-de-cuidados-prolongados-triplicara-nas-americas-ate-2050

3. Minayo MCS. O imperativo de cuidar da pessoa idosa dependente. Cien Saude Colet 2019; 24(1):247-252.

4. Organização Pan-Americana da Saúde (OPAS). Agenda de saúde sustentável para as Américas 2018-2030: um chamado à ação para a saúde e o bem-estar na região [matéria na Internet]. Washington: OPAS; 2017 [acessado 2019 Fev 27]. Disponível em: https://www.paho. org/hq/index.php?option=com_docman\&task $=$ doc download\&gid $=42117 \&$ Itemid $=270 \&$ lang $=p t$

5. Campos ACV, Goncalves LHT. Perfil demográfico do envelhecimento nos municípios do Estado do Pará, Brasil. Rev Bras Enferm 2018; 71(Supl.1):591-598.

6. Brasil. Lei n. 8.842, de 4 de janeiro de 1994. Dispõe sobre a Política Nacional do Idoso, cria o Conselho Nacional do Idoso e dá outras providências. Diário Oficial da União 1994; 5 jan.

7. Brasil. Lei n. 10.741, de $1^{\circ}$ de outubro de 2003. Dispõe sobre o Estatuto do Idoso e dá outras providências. Diário Oficial da União 2003; 3 out.

8. Brasil. Ministério da Saúde (MS). Portaria n. 2.528, de 19 de outubro de 2006. Aprova a Política Nacional de Saúde da Pessoa Idosa. Diário Oficial da União 2006; 20 out.

9. Brasil. Ministério da Saúde (MS). Portaria n. 648, de 28 de março de 2006. Dispõe sobre a aprovação da Política de Atenção Básica, estabelecendo diretrizes e normas para a organização da Atenção Básica para o Programa Saúde da Família (PSF) e o Programa de Agentes Comunitários de Saúde (PACS). Diário Oficial da União 2006; 29 mar.

10. Brasil. Ministério da Saúde (MS). Plano de ações estratégicas para o enfrentamento das doenças crônicas não transmissíveis (DCNT) no Brasil 2011-2022. Brasília: MS; 2011.

11. Brasil. Decreto n. 8.114, de 30 de setembro de 2013. Estabelece o compromisso nacional para o envelhecimento ativo e institui comissão interministerial para monitorar e avaliar ações em seu âmbito e promover a articulação de órgãos e entidades públicos envolvidos em sua implementação. Diário Oficial da União 2013; 1 out.

12. Brasil. Ministério da Saúde (MS). Orientações técnicas para a implementação de Linha de Cuidado para Atenção Integral à Saúde da Pessoa Idosa no Sistema Único de Saúde - SUS [livro na Internet]. Brasília: MS; 2018. [acessado 2020 Maio 05]. Disponível em: http:// bvsms.saude.gov.br/bvs/publicacoes/linha_cuidado_ atencao_pessoa_idosa.pdf
13. Cecilio LCO. Apontamentos teórico-conceituais sobre processos avaliativos considerando as múltiplas dimensões da gestão do cuidado em saúde. Interface (Botucatu) 2011; 15(37):598-599.

14. Schenker M, Costa DH. Avanços e desafios da atenção à saúde da população idosa com doenças crônicas na Atenção Primária à Saúde. Cien Saude Colet [periódico na Internet]. 2019 Abr [acessado 2020 Maio 17]; 24(4):1369-1380. Disponível em: https://www.scielo.br/pdf/csc/v24n4/1413-8123csc-24-04-1369.pdf

15. Oliveira MR, Veras RP, Cordeiro HA. A importância da porta de entrada no sistema: o modelo integral de cuidado para o idoso. Physis [periódico na Internet]. 2019 [acessado 2020 Maio 05]; 28(4):e280411. Disponível em: https://www.scielo.br/pdf/physis/ v28n4/0103-7331-physis-28-04-e280411.pdf

16. Habermas J, Redondo MJ. Teoría de la acción comunicativa. Madrid: Taurus; 1987.

17. Minayo MCS. O desafio do conhecimento: pesquisa qualitativa em saúde. 13a ed. São Paulo: Hucitec; 2013.

18. Barreto ML. Desigualdades em Saúde: uma perspectiva global. Cien Saude Colet [periódico na Internet]. 2017 Julho [acessado 2020 Maio 21]; 22(7):20972108. Disponível em: https://www.scielo.br/pdf/csc/ v22n7/1413-8123-csc-22-07-2097.pdf

19. Brasil. Conselho Nacional de Saúde (CNS). Resolução $n^{\circ} 466$, de 12 de dezembro de 2012. Dispõe das normas para pesquisa envolvendo seres humanos. Diário Oficial União 2013; 13 jun.

20. Ravioli AF, Soarez PC, Scheffer MC. Modalidades de gestão de serviços no Sistema Único de Saúde: revisão narrativa da produção científica da Saúde Coletiva no Brasil (2005-2016). Cad Saude Publica 2018; 34(4):e00114217.

21. Martins CC, Waclawovsky AJ. Problemas e desafios enfrentados pelos gestores públicos no processo de gestão em saúde. Rev Gest Sist Saúde 2015; 4(1):100109.

22. Leite MT, Nardino J, Hildebrandt LM, Santos AM, Martins RV. Gestão do cuidado na estratégia saúde da família: revisão narrativa. Rev Aten Saude 2016; 14(48):106-115.

23. Silva SS, Assis MMA, Santos AM. The nurse as the protagonist of care management in the Estratégia Saúde da Família: different analysis perspectives. Texto \& Contexto Enferm 2017; 26(3):e1090016.

24. Veras RP, Oliveira M. Envelhecer no Brasil: a construção de um modelo de cuidado. Cien Saude Colet 2018; 23(6):1929-1936.

25. Poças KC, Freitas, LRS, Duarte EC. Censo de estrutura da Atenção Primária à Saúde no Brasil (2012): estimativas de coberturas potenciais. Epidemiol Serv Saúde [periódico na Internet]. 2017 [acessado 2020 Maio 14]; 26(2):275-284. Disponível em: https://www.scielo.br/pdf/ress/v26n2/2237-9622-ress-26-02-00275. pdf

26. Almeida PF, Medina MG, Fausto MCR, Giovanella L, Bousquat A, Mendonça MHM. Coordenação do cuidado e Atenção Primária à Saúde no Sistema Único de Saúde. Saúde debate [periódico na Internet]. 2018 Set [acessado 2020 Junho 05]; 42(n. esp. 1):244-260. Disponível em: http://www.scielo.br/scielo.php?script=sci_arttext\&pid=S0103-11042018000500244\&lng=pt 
27. Lima CA, Moreira KS, Barbosa BCS, Souza Júnior RL, Pinto MQC, Costa SM. Atenção integral à comunidade: autoavaliação das equipes de saúde da família. Av Enferm [periódico na Internet]. 2019 [acessado 2020 Maio 21]; 37(3):303-312. Disponível em: http://www.scielo.org.co/pdf/aven/v37n3/0121-4500aven-37-03-303.pdf

28. Kantorski LP, Coimbra VCC, Oliveira NA, Nunes CK, Pavani FM, Sperb LCSO. Atenção psicossocial infantojuvenil: interfaces com a rede de saúde pelo sistema de referência e contrarreferência. Texto \& Contexto Enferm 2017; 26(3):e1890014.

29. Sturbelle ICS, Pai DD, Tavares JP, Trindade LL, Riquinho DL, Ampos LF. Violência no trabalho em saúde da família: estudo de métodos mistos. Acta Paul Enferm 2019; 32(6):632-641.

30. Souza ER, Minayo MCS. Segurança pública num país violento. Cad Saude Publica [periódico na Internet]. 2017[acessado 2020 Maio 14]; 33(3):e00036217. Disponível em: https://www.scielo.br/pdf/csp/v33n3/ 1678-4464-csp-33-03-e00036217.pdf

31. Budib MB, Zulim MI, Oliveira VM, Matos VTG. Integrated continuous care: collaborating with the elderly functionality. Biosci J 2020; 36(1):266-275.

32. Chen C, Huang Y, Liu C, Xu Y, Zheng L, Li J. Effects of an interdisciplinary care team on the management of alzheimer's disease in China. J Gerontol Nursing 2019; 45(5):39-45.

33. Santos SC, Tonhom SFR, Komatsu RS. Saúde do idoso: reflexões acerca da integralidade do cuidado. Rev Bras Promoç Saúde 2016; 29:118-127.

34. Santos DS, Mishima SM, Merhy EE. Processo de trabalho na Estratégia de Saúde da Família: potencialidades da subjetividade do cuidado para reconfiguração do modelo de atenção. Cien Saude Colet [periódico na Internet]. 2018 Mar [acessado 2020 Maio 21]; 23(3):861-870. Disponível em: https://www.scielo.br/ pdf/csc/v23n3/1413-8123-csc-23-03-0861.pdf

35. Xavier GTO, Nascimento VB, Carneiro N Jr. Atenção domiciliar e sua contribuição para a construção das Redes de Atenção à Saúde sob a óptica de seus profissionais e de usuários idosos. Rev Bras Geriatr Gerontol [periódico na Internet]. 2019 [acessado 2020 May 17]; 22(2):e180151. Disponível em: https://www.scielo.br/ pdf/rbgg/v22n2/pt_1809-9823-rbgg-22-02-e180151. pdf

36. Rissardo LK, Carreira L. Organization of healthcare and assistance to the elderly indigenous population: synergies and particularities of the professional context. Rev Esc Enferm USP 2014; 48(1):72-79.

37. Wen T, Zhang Y, Wang X, Tang G. Factors influencing turnover intention among primary care doctors: a cross-sectional study in Chongqing, China. Hum Resour Health 2018; 16:10.

38. Cabral R, Gomes Dellaroza MS, Carvalho BG, Zani AV. Elderly care in primary health care from the perspective of health professionals. Cienc Cuid Saúde [serial on Internet] 2019 [acessado 2020 Maio 17];18(2):e450261. Disponível em: http://periodicos.uem.br/ojs/index.php/CiencCuidSaude/article/ view/45026/pdf_1
39. Baptistini APF, Zanon CJ. O sistema de informação na gestão do cuidado: estudo de caso em instituição de longa permanência para idosos. Estud Interdiscip Envelhec 2019; 24(1):63-74.

40. Rocha FCV, Ferreira BR, Silva FP, Oliveira ADS, Amorim FCM. Elderly welcoming in primary health care: the user perspective. Rev Pesqui 2018; 10(3):669-674.

41. Brasil. Ministério da Saúde (MS). HumanizaSUS: política nacional de humanização. Brasília: MS; 2004.

42. Sacco RCCS, Cardoso PRR, Escalda PMF, Assis MG, Guimarães SMF. Avaliação da microgestão em Unidades Básicas de Saúde em ações para idosos em uma região de saúde do Distrito Federal, Brasil. Cien Saude Colet [periódico na Internet]. 2019 Jun [acessado 2020 Maio 17]; 24(6):2173-2183. Disponível em: https://www.scielo.br/pdf/csc/v24n6/1413-8123csc-24-06-2173.pdf

43. Araújo LF, Castro JLC, Santos, JVO. A família e sua relação com o idoso: um estudo de representações sociais. Psicol Pesq 2018; 12(2):14-23.

44. Suriyawongpaisal P, Aekplakorn W, Leerapan B, Lakha F, Srithamrongsawat S, von Bormann S. Assessing system-based trainings for primary care teams and quality-of-life of patients with multimorbidity in Thailand: patient and provider surveys. BMC Fam Pract 2019; 20(1):85.

45. Iwasaki R, Hirai K, Kageyama T, Satoh T, Fukuda H, Kai H, Makino K, Magilvy K, Murashima S. Supporting elder persons in rural Japanese communities through preventive home visits by nursing students: a qualitative descriptive analysis of students' reports. Public Health Nurs 2019; 36(4):557-563.

46. Akhtar S, Loganathan M, Nowaczynski M, Sinha S, Condon A, Ewa V, Kirk JC, Pham T-N. Aging at home: a portrait of home-based primary care across Canada. Healthc Q 2019; 22(1):30-35.

Artigo apresentado em 15/06/2020

Aprovado em 21/08/2020

Versão final apresentada em 23/08/2020

Editores chefes: Maria Cecília de Souza Minayo, Romeu Gomes, Antônio Augusto Moura da Silva. Editora Associada da área de Saúde do Idoso: Joselia Oliveira Araújo Firmo 